\title{
ON THE PRODUCT OF PROJECTORS AND GENERALIZED INVERSES
}

\author{
ANJA KORPORAL AND GEORG REGENSBURGER
}

\begin{abstract}
We consider generalized inverses of linear operators on arbitrary vector spaces and study the question when their product in reverse order is again a generalized inverse. This problem is equivalent to the question when the product of two projectors is again a projector, and we discuss necessary and sufficient conditions in terms of their kernels and images alone. We give a new representation of the product of generalized inverses that does not require explicit knowledge of the factors. Our approach is based on implicit representations of subspaces via their orthogonals in the dual space. For Fredholm operators, the corresponding computations reduce to finite-dimensional problems. We illustrate our results with examples for matrices and linear ordinary boundary problems.
\end{abstract}

\section{INTRODUCTION}

Analogues of the reverse order law $(A B)^{-1}=B^{-1} A^{-1}$ for bijective operators have been studied intensively for various kinds of generalized inverses. Most articles and books are concerned with the matrix case; see for example [9, 8, 19, 23, 29, 4, 2, 16, 26, 27, 15. For infinite-dimensional vector spaces, usually additional topological structures like Banach or Hilbert spaces are assumed; see for example 17, 7, 6, 5. In our approach, we systematically exploit duality results that hold in arbitrary vector spaces and a corresponding duality principle for statements about generalized inverses and projectors; see Appendix (A).

The validity of the reverse order law can be reduced to the question whether the product of two projectors is a projector (Section 2). This problem is studied in [11, 25, 28, for finite-dimensional vector spaces. We discuss necessary and sufficient conditions that carry over to arbitrary vector spaces and can be expressed in terms of the kernels and images of the respective operators alone (Section 4). Applying the duality principle leads to new conditions and a characterization of the commutativity of two projectors that generalizes a result from [1.

In Section 5 we translate the results for projectors to generalized inverses and obtain necessary and sufficient conditions for the reverse order law in arbitrary vector spaces. Based on these conditions, we give a short proof for the characterization in Theorem 12 of two operators such that the reverse holds for all inner inverses (also called g-inverses or $\{1\}$-inverses). Moreover, we show that there always exist algebraic generalized inverses (also called $\{1,2\}$-inverses) of two operators $A$ and $B$ such that their product in reverse order is an algebraic generalized inverse of $A B$.

Date: August 14, 2013.

2000 Mathematics Subject Classification. Primary 15A09; Secondary 47A05.

Key words and phrases. Generalized inverse, projector, reverse order law, Fredholm operator, linear boundary problem, duality. 
Assuming the reverse order law to hold, Theorem 15 gives a representation of the product of two outer inverses $(\{2\}$-inverses) that can be computed using only kernel and image of the outer inverses of the factors. In this representation, we rely on a description of the kernel of a composition using inner inverses (Section 3) and implicit representations of subspaces via their orthogonals in the dual space. Moreover, we avoid the computation of generalized inverses by using the associated transpose map. Examples for matrices illustrating the results are given in Section 7

An important application for our results is given by linear boundary problems (Section 9). Their solution operators (Green's operators) are generalized inverses, and it is natural to express infinite dimensional solution spaces implicitly via the (homogeneous) boundary conditions they satisfy. Green's operators for ordinary boundary problems are Fredholm operators, for which we can check the conditions for the reverse order law algorithmically and compute the implicit representation of the product (Section 8). Hence we can test if the product of two (generalized) Green's operators is again a Green's operator, and we can determine which boundary problem it solves.

\section{Generalized inverses}

In this section, we first recall basic properties of generalized inverses. For further details and proofs, we refer to [18, 17] and the references therein. Throughout this article, $U, V$, and $W$ always denote vector spaces over the same field $F$, and we use the notation $V_{1} \leq V$ for a subspace $V_{1}$ of $V$.

Definition 1. Let $T: V \rightarrow W$ be linear. We call a linear map $G: W \rightarrow V$ an inner inverse of $T$ if $T G T=T$ and an outer inverse of $T$ if $G T G=G$. If $G$ is an inner and an outer inverse of $T$, we call $G$ an algebraic generalized inverse of $T$.

This terminology of generalized inverses is adopted from [18; other sources refer to inner inverses as generalized inverses or g-inverses, whereas algebraic generalized inverses are also called reflexive generalized inverses. Also the notations $\{1\}$-inverse (resp. $\{2\}$ - and $\{1,2\}$-inverse) are used, which refer to the corresponding MoorePenrose equations the generalized inverse satisfies.

Proposition 2. Let $T: V \rightarrow W$ and $G: W \rightarrow V$ be linear. The following statements are equivalent:

(i) $G$ is an outer inverse of $T$.

(ii) $G T$ is a projector and $\operatorname{Im} G T=\operatorname{Im} G$.

(iii) $G T$ is a projector and $V=\operatorname{Im} G \oplus \operatorname{Ker} G T$.

(iv) $G T$ is a projector and $W=\operatorname{Im} T+\operatorname{Ker} G$.

(v) $T G$ is a projector and $\operatorname{Ker} T G=\operatorname{Ker} G$.

(vi) $T G$ is a projector and $W=\operatorname{Ker} G \oplus \operatorname{Im} T G$.

(vii) $T G$ is a projector and $\operatorname{Im} G \cap \operatorname{Ker} T=\{0\}$.

Corresponding to (vii) and (vi) for subspaces $B \leq V$ and $E \leq W$ with

$$
B \cap \operatorname{Ker} T=\{0\} \quad \text { and } \quad W=E \oplus T(B),
$$

we can construct an outer inverse $G$ of $T$ with $\operatorname{Im} G=B$ and $\operatorname{Ker} G=E$ as follows; cf. [17, Cor. 8.2]. We consider the projector $Q$ with

$$
\operatorname{Im} Q=T(B), \quad \operatorname{Ker} Q=E .
$$


The restriction $\left.T\right|_{B}: B \rightarrow T(B)$ is bijective since $B \cap \operatorname{Ker} T=\{0\}$, and we can define $G=\left(\left.T\right|_{B}\right)^{-1} Q$. One easily verifies that $G$ is an outer inverse of $T$ with $\operatorname{Im} G=B$ and $\operatorname{Ker} G=E$. Since by (iii) we have $V=B \oplus T^{-1}(E)$, we define the projector $P$ in analogy to $Q$ by

$$
\operatorname{Im} P=T^{-1}(E), \text { Ker } P=B .
$$

Then, by definition and by Proposition 2, we have

$$
G T G=G, \quad T G=Q, \quad \text { and } \quad G T=1-P,
$$

and $G$ is determined uniquely by these equations. Hence an outer inverse depends only on the choice of the defining spaces $B$ and $E$. We use the notations $G=$ $\mathrm{O}(T, B, E)$ and $G=\mathrm{O}(T, P, Q)$ for $P$ and $Q$ as in (2) and (1).

Obviously, $G$ is an outer inverse of $T$ if and only if $T$ is an inner inverse of $G$. Therefore, we get a result analogous to Proposition 2 for inner inverses by interchanging the role of $T$ and $G$. The construction of inner inverses is not completely analogous to outer inverses, see [18, Prop. 1.3]. For subspaces $B \leq V$ and $E \leq W$ such that

$$
V=\operatorname{Ker} T \oplus B \quad \text { and } \quad W=\operatorname{Im} T \oplus E,
$$

an inner inverse $G$ of $T$ is given on $\operatorname{Im} T$ by $\left(\left.T\right|_{B}\right)^{-1}$ and can be chosen arbitrarily on $E$. For such an inner inverse with $B=\operatorname{Im} G T$ and $E=\operatorname{Ker} T G$, we write $G \in \mathrm{I}(T, B, E)$.

For constructing algebraic generalized inverses, we start with direct sums as in (3), but require $\operatorname{Ker} G=E$ and $\operatorname{Im} G=B$. We use the notation $G=\mathrm{G}(T, B, E)$.

The following result for inner inverses is well-known in the matrix case [22, 23, 28 , and its elementary proof remains valid for arbitrary vector spaces.

Proposition 3. Let $T_{1}: V \rightarrow W$ and $T_{2}: U \rightarrow V$ be linear with outer (resp. inner) inverses $G_{1}$ and $G_{2}$. Let $P=G_{1} T_{1}$ and $Q=T_{2} G_{2}$. Then $G_{2} G_{1}$ is an outer (resp. inner) inverse of $T_{1} T_{2}$ if and only if $Q P$ (resp. $P Q$ ) is a projector.

Proof. Let $G_{2} G_{1}$ be an outer inverse of $T_{1} T_{2}$, that is, $G_{2} G_{1}=G_{2} G_{1} T_{1} T_{2} G_{2} G_{1}$. Multiplying with $T_{2}$ from the left and with $T_{1}$ from the right yields

$$
T_{2} G_{2} G_{1} T_{1}=T_{2} G_{2} G_{1} T_{1} T_{2} G_{2} G_{1} T_{1},
$$

thus $Q P=T_{2} G_{2} G_{1} T_{1}$ is a projector. For the other direction, we multiply the previous equation with $G_{2}$ from the left and $G_{1}$ from the right and use that $G_{1} T_{1} G_{1}=G_{1}$ and $G_{2} T_{2} G_{2}=G_{2}$. The proof for inner inverses follows by interchanging the roles of $T_{i}$ and $G_{i}$.

\section{KERNEL OF COMPOSITIONS}

We now describe the inverse image of a subspace under the composition of two linear maps using inner inverses. For projectors, kernel and image of the composition can be expressed in terms of kernel and image of the corresponding factors alone. Note that a projector is an inner inverse of itself.

Proposition 4. Let $T_{1}: V \rightarrow W$ and $T_{2}: U \rightarrow V$ be linear and $G_{2}$ an inner inverse of $T_{2}$. For a subspace $W_{1} \leq W$, we have

$$
\left(T_{1} T_{2}\right)^{-1}\left(W_{1}\right)=G_{2}\left(T_{1}^{-1}\left(W_{1}\right) \cap \operatorname{Im} T_{2}\right) \oplus \operatorname{Ker} T_{2}
$$


for the inverse image of the composition. In particular,

$$
\operatorname{Ker} T_{1} T_{2}=G_{2}\left(\operatorname{Ker} T_{1} \cap \operatorname{Im} T_{2}\right) \oplus \operatorname{Ker} T_{2} .
$$

Proof. Since $T_{2} G_{2}$ is a projector onto $\operatorname{Im} T_{2}$ by Proposition 2 (ii) (interchanging the role of $T$ and $G$ ), we have

$$
\begin{aligned}
T_{1} T_{2}\left(G_{2}\left(T_{1}^{-1}\left(W_{1}\right) \cap \operatorname{Im} T_{2}\right)+\right. & \left.\operatorname{Ker} T_{2}\right)=T_{1} Q_{2}\left(T_{1}^{-1}\left(W_{1}\right) \cap \operatorname{Im} T_{2}\right)+0 \\
= & T_{1}\left(T_{1}^{-1}\left(W_{1}\right) \cap \operatorname{Im} T_{2}\right) \leq W_{1} \cap \operatorname{Im} T_{1} T_{2} \leq W_{1} .
\end{aligned}
$$

Conversely, let $u \in\left(T_{1} T_{2}\right)^{-1}\left(W_{1}\right)$. Then $T_{2} u=v$ with $v \in T_{1}^{-1}\left(W_{1}\right)$. Since also $v \in \operatorname{Im} T_{2}$, we have

$$
T_{2}\left(u-G_{2} v\right)=T_{2} u-Q_{2} v=T_{2} u-v=v-v=0,
$$

that is, $u-G_{2} v \in \operatorname{Ker} T_{2}$. Writing $u=G_{2} v+u-G_{2} v$ yields $u \in G_{2}\left(T_{1}^{-1}\left(W_{1}\right) \cap\right.$ $\left.\operatorname{Im} T_{2}\right)+\operatorname{Ker} T_{2}$. The sum is direct since by Proposition 2 (vi) (interchanging the role of $T$ and $G$ ), we have $U=\operatorname{Ker} T_{2} \oplus \operatorname{Im} G_{2} T_{2}$.

Corollary 5. Let $T: V \rightarrow W$ be linear and let $P: V \rightarrow V$ and $Q: W \rightarrow W$ be projectors. Then

$\operatorname{Ker} T Q=(\operatorname{Ker} T \cap \operatorname{Im} Q) \oplus \operatorname{Ker} Q \quad$ and $\quad \operatorname{Im} P T=(\operatorname{Im} T+\operatorname{Ker} P) \cap \operatorname{Im} P$.

Proof. Applying Proposition 4 yields

$$
\operatorname{Ker} T Q=Q(\operatorname{Ker} T \cap \operatorname{Im} Q) \oplus \operatorname{Ker} Q=(\operatorname{Ker} T \cap \operatorname{Im} Q) \oplus \operatorname{Ker} Q .
$$

The statement for the image follows from the duality principle 22.

This result generalizes [28, Lemma 2.2], where the kernel and image of a product $P Q$ of two projectors are computed as above, when $P Q$ is again a projector.

\section{Products of PRojectors}

In view of Proposition 3, we study necessary and sufficient conditions for the product of two projectors to be a projector. Throughout this section let $P, Q: V \rightarrow$ $V$ denote projectors.

The first of the following necessary and sufficient conditions for the product of $P$ and $Q$ to be a projector is mentioned as an exercise without proof in [3, p. 339]. In 11, Lemma 3] the same result is formulated for matrices but the proof is valid for arbitrary vector spaces. The second necessary and sufficient condition for the matrix case is given in [28, Lemma 2.2]. The simpler proof from [25] carries over to arbitrary vector spaces.

Lemma 6. The composition $P Q$ is a projector if and only if

$$
\operatorname{Im} P Q \leq \operatorname{Im} Q \oplus(\operatorname{Ker} P \cap \operatorname{Ker} Q)
$$

if and only if

$$
\operatorname{Im} Q \leq \operatorname{Im} P \oplus(\operatorname{Ker} P \cap \operatorname{Im} Q) \oplus(\operatorname{Ker} P \cap \operatorname{Ker} Q) .
$$

We obtain the following characterization of the idempotency of $P Q$ in terms of the kernels and images of $P$ and $Q$ alone.

Theorem 7. The following statements are equivalent:

(i) The composition $P Q$ is a projector.

(ii) $\operatorname{Im} P \cap(\operatorname{Im} Q+\operatorname{Ker} P) \leq \operatorname{Im} Q \oplus(\operatorname{Ker} P \cap \operatorname{Ker} Q)$ 
(iii) $\operatorname{Im} Q \leq \operatorname{Im} P \oplus(\operatorname{Ker} P \cap \operatorname{Im} Q) \oplus(\operatorname{Ker} P \cap \operatorname{Ker} Q)$

(iv) $\operatorname{Ker} Q \oplus(\operatorname{Ker} P \cap \operatorname{Im} Q) \geq \operatorname{Ker} P \cap(\operatorname{Im} Q+\operatorname{Im} P)$

(v) $\operatorname{Ker} P \geq \operatorname{Ker} Q \cap(\operatorname{Im} Q+\operatorname{Ker} P) \cap(\operatorname{Im} Q+\operatorname{Im} P)$

Proof. The equivalence of (i), (ii), (iii) follow from the previous lemma and Corollary [5. By the duality principle [22, the last two conditions are equivalent to (ii) and (iii), respectively.

For algebraic generalized inverses, it is also interesting to have sufficient conditions for $P Q$ as well as $Q P$ to be projectors; for example, if $P$ and $Q$ commute. This can again be characterized in terms of the images and kernels of $P$ and $Q$ alone. If $P Q=Q P$, one sees with Corollary 5 that

$$
\operatorname{Im} P Q=\operatorname{Im} P \cap \operatorname{Im} Q \quad \text { and } \quad \operatorname{Ker} P Q=\operatorname{Ker} P+\operatorname{Ker} Q .
$$

In general, these conditions are necessary but not sufficient for commutativity of $P$ and $Q$, see [11, Ex. 1].

Using Corollary 5 modularity (8), and (9), one obtains the following characterization of projectors with image or kernel as in (4); for further details see [12. For the commutativity of projectors see also [3, p. 339].

Proposition 8. The composition $P Q$ is a projector with

(i) $\operatorname{Im} P Q=\operatorname{Im} P \cap \operatorname{Im} Q$ if and only if

$$
\operatorname{Im} Q=(\operatorname{Im} P \cap \operatorname{Im} Q) \oplus(\operatorname{Ker} P \cap \operatorname{Im} Q) .
$$

(ii) $\operatorname{Ker} P Q=\operatorname{Ker} P+\operatorname{Ker} Q$ if and only if

$$
\text { Ker } P=(\operatorname{Ker} P \cap \operatorname{Ker} Q) \oplus(\operatorname{Ker} P \cap \operatorname{Im} Q) \text {. }
$$

Corollary 9. We have $P Q=Q P$ if and only if

$$
\operatorname{Im} Q=(\operatorname{Im} P \cap \operatorname{Im} Q) \oplus(\operatorname{Ker} P \cap \operatorname{Im} Q)
$$

and

$$
\operatorname{Ker} Q=(\operatorname{Im} P \cap \operatorname{Ker} Q) \oplus(\operatorname{Ker} P \cap \operatorname{Ker} Q) .
$$

In [11, Thm. 4] and [1, Thm. 3.2] different necessary and sufficient conditions for the commutativity of two projectors are given, but both require the computation of $P Q$ as well as of $Q P$.

\section{REVERSE ORDER LAW FOR GENERALIZED INVERSES}

Proposition 3 and Theorem 7 together give necessary and sufficient conditions for the reverse order law for outer inverses to hold, in terms of the defining spaces $B_{i}$ and $E_{i}$ alone.

Theorem 10. Let $T_{1}: V \rightarrow W$ and $T_{2}: U \rightarrow V$ be linear with outer inverses $G_{1}=$ $\mathrm{O}\left(T_{1}, B_{1}, E_{1}\right)$ and $G_{2}=\mathrm{O}\left(T_{2}, B_{2}, E_{2}\right)$. The following conditions are equivalent:

(i) $G_{2} G_{1}$ is an outer inverse of $T_{1} T_{2}$.

(ii) $T_{2}\left(B_{2}\right) \cap\left(B_{1}+E_{2}\right) \leq B_{1} \oplus\left(E_{2} \cap T_{1}^{-1}\left(E_{1}\right)\right)$

(iii) $B_{1} \leq T_{2}\left(B_{2}\right) \oplus\left(E_{2} \cap B_{1}\right) \oplus\left(E_{2} \cap T_{1}^{-1}\left(E_{1}\right)\right)$

(iv) $T_{1}^{-1}\left(E_{1}\right) \oplus\left(E_{2} \cap B_{1}\right) \geq E_{2} \cap\left(B_{1}+T_{2}\left(B_{2}\right)\right)$

(v) $E_{2} \geq T_{1}^{-1}\left(E_{1}\right) \cap\left(B_{1}+E_{2}\right) \cap\left(B_{1}+T_{2}\left(B_{2}\right)\right)$ 
Proof. Recall that $\operatorname{Im} G_{i}=B_{i}$ and $\operatorname{Ker} G_{i}=E_{i}$, and $Q=T_{2} G_{2}$ and $P=G_{1} T_{1}$ are projectors with

$$
\operatorname{Im} P=B_{1}, \quad \operatorname{Ker} P=T_{1}^{-1}\left(E_{1}\right), \quad \operatorname{Im} Q=T_{2}\left(B_{2}\right), \quad \text { and } \quad \operatorname{Ker} Q=E_{2} .
$$

By Proposition $3, G_{2} G_{1}$ is an outer inverse if and only if $Q P$ is a projector. Applying Theorem 7 proves the claim.

In the following theorem, we give the analogous conditions for inner inverses, where $P=G_{1} T_{1}$ and $Q=T_{2} G_{2}$ are the projectors corresponding to the direct sums in (3). Note that the conditions for inner inverses only depend on the choice of $B_{1}$ and $E_{2}$, but not on $B_{2}$ and $E_{1}$.

The characterization (iii) and the orthogonal of (v) in the following theorem generalize [28, Thm. 2.3] to arbitrary vector spaces.

Theorem 11. Let $T_{1}: V \rightarrow W$ and $T_{2}: U \rightarrow V$ be linear with inner inverses $G_{1} \in \mathrm{I}\left(T_{1}, B_{1}, E_{1}\right)$ and $G_{2} \in \mathrm{I}\left(T_{2}, B_{2}, E_{2}\right)$. The following conditions are equivalent:

(i) $G_{2} G_{1}$ is an inner inverse of $T_{1} T_{2}$.

(ii) $B_{1} \cap\left(\operatorname{Im} T_{2}+\operatorname{Ker} T_{1}\right) \leq \operatorname{Im} T_{2} \oplus\left(\operatorname{Ker} T_{1} \cap E_{2}\right)$

(iii) $\operatorname{Im} T_{2} \leq B_{1} \oplus\left(\operatorname{Ker} T_{1} \cap \operatorname{Im} T_{2}\right) \oplus\left(\operatorname{Ker} T_{1} \cap E_{2}\right)$

(iv) $E_{2} \oplus\left(\operatorname{Ker} T_{1} \cap \operatorname{Im} T_{2}\right) \geq \operatorname{Ker} T_{1} \cap\left(\operatorname{Im} T_{2}+B_{1}\right)$

(v) $\operatorname{Ker} T_{1} \geq E_{2} \cap\left(\operatorname{Im} T_{2}+\operatorname{Ker} T_{1}\right) \cap\left(\operatorname{Im} T_{2}+B_{1}\right)$

The question when the reverse order law holds for all inner inverses of $T_{1}$ and $T_{2}$ was answered for matrices in [29, Thm. 2.3], and an alternative proof was given in [10. Using the previous characterizations, we give a short proof that generalizes the result to arbitrary vector spaces.

Theorem 12. Let $T_{1}: V \rightarrow W$ and $T_{2}: U \rightarrow V$ be linear. Then $G_{2} G_{1}$ is an inner inverse of $T_{1} T_{2}$ for all inner inverses $G_{1}$ of $T_{1}$ and $G_{2}$ of $T_{2}$ if and only if $T_{1} T_{2}=0$ or $\operatorname{Ker} T_{1} \leq \operatorname{Im} T_{2}$.

Proof. If $\operatorname{Ker} T_{1} \leq \operatorname{Im} T_{2}$ then $\operatorname{Ker} T_{1} \cap \operatorname{Im} T_{2}=\operatorname{Ker} T_{1}$ and (iii) in the previous theorem is satisfied since $\operatorname{Ker} T_{1}+B_{1}=V$. The case $T_{1} T_{2}=0$ is trivial.

For the reverse implication, assume that $\operatorname{Im} T_{2}$ is not contained in $\operatorname{Ker} T_{1}$ and $\operatorname{Ker} T_{1}$ is not contained in $\operatorname{Im} T_{2}$. Choose $V_{1}, V_{2} \leq V$ such that we have two direct sums $\operatorname{Ker} T_{1}=\left(\operatorname{Im} T_{2} \cap \operatorname{Ker} T_{1}\right) \oplus V_{1}$ and $\operatorname{Im} T_{2}=\left(\operatorname{Im} T_{2} \cap \operatorname{Ker} T_{1}\right) \oplus V_{2}$. Then we have

$$
\operatorname{Im} T_{2}+\operatorname{Ker} T_{1}=\left(\operatorname{Im} T_{2} \cap \operatorname{Ker} T_{1}\right) \oplus V_{1} \oplus V_{2} .
$$

By assumption, we can choose non-zero $v_{1} \in V_{1}$ and $v_{2} \in V_{2}$. Let $v=v_{1}+v_{2}$. Then $v \in \operatorname{Im} T_{2}+\operatorname{Ker} T_{1}$ and $v \notin \operatorname{Ker} T_{1}, v \notin \operatorname{Im} T_{2}$. Hence we can choose $B_{1}$ and $E_{2}$ such that $v \in B_{1}$ and $v \in E_{2}$ and $V=\operatorname{Ker} T_{1} \oplus B_{1}=\operatorname{Im} T_{2} \oplus E_{2}$. Then

$$
v \in E_{2} \cap\left(\operatorname{Im} T_{2}+\operatorname{Ker} T_{1}\right) \cap\left(\operatorname{Im} T_{2}+B_{1}\right)
$$

but $v \in \operatorname{Ker} T_{1}$. Hence (v) in the previous theorem is not satisfied for inner inverses with $\operatorname{Im} G_{1}=B_{1}$ and $\operatorname{Ker} G_{2}=E_{2}$.

Werner [28, Thm. 3.1] proves that for matrices it is always possible to construct inner inverses such that the reverse order law holds. Using the necessary and sufficient condition for outer inverses above, we extend this result to algebraic generalized inverses in arbitrary vector spaces. The special case of Moore-Penrose inverses is treated in [23, Thm. 3.2], and explicit solutions are constructed in [24, 30]. 
Theorem 13. Let $T_{1}: V \rightarrow W$ and $T_{2}: U \rightarrow V$ be linear. There always exist algebraic generalized inverses $G_{1}$ of $T_{1}$ and $G_{2}$ of $T_{2}$ such that $G_{2} G_{1}$ is an algebraic generalized inverse of $T_{1} T_{2}$.

Proof. Choose $V_{1}, V_{2} \leq V$ as in the previous proof such that (5) holds. Moreover, choose $V_{3} \leq V$ such that

$$
V=\left(\operatorname{Im} T_{2}+\operatorname{Ker} T_{1}\right) \oplus V_{3}=\left(\operatorname{Im} T_{2} \cap \operatorname{Ker} T_{1}\right) \oplus V_{1} \oplus V_{2} \oplus V_{3} .
$$

Then $B_{1}=V_{2} \oplus V_{3}$ is a direct complement of $\operatorname{Ker} T_{1}$, and $E_{2}=V_{1} \oplus V_{3}$ is a direct complement of $\operatorname{Im} T_{2}$. Hence there exist respectively an algebraic generalized inverse $G_{1}$ of $T_{1}$ with $\operatorname{Im} G_{1}=B_{1}$ and $G_{2}$ of $T_{2}$ with $\operatorname{Ker} G_{2}=E_{2}$. We verify that such $G_{1}$ and $G_{2}$ satisfy Theorem 10 (iii), where $T_{1}^{-1}\left(E_{1}\right)=\operatorname{Ker} T_{1}$ and $T_{2}\left(B_{2}\right)=\operatorname{Im} T_{2}$ since $G_{1}$ and $G_{2}$ are algebraic generalized inverses:

$$
\operatorname{Im} T_{2} \oplus\left(E_{2} \cap B_{1}\right) \geq \operatorname{Im} T_{2} \oplus V_{3}=\left(\operatorname{Im} T_{2} \cap \operatorname{Ker} T_{1}\right) \oplus V_{2} \oplus V_{3} \geq B_{1} .
$$

Similarly, we verify Theorem 11 (iii)

$B_{1} \oplus\left(\operatorname{Ker} T_{1} \cap \operatorname{Im} T_{2}\right)=V_{2} \oplus V_{3} \oplus\left(\operatorname{Ker} T_{1} \cap \operatorname{Im} T_{2}\right) \geq V_{2} \oplus\left(\operatorname{Ker} T_{1} \cap \operatorname{Im} T_{2}\right)=\operatorname{Im} T_{2}$.

Hence $G_{2} G_{1}$ is an algebraic generalized inverse of $T_{1} T_{2}$ for all $G_{1}=\mathrm{G}\left(T_{1}, B_{1}, E_{1}\right)$ and $G_{2}=\mathrm{G}\left(T_{2}, B_{2}, E_{2}\right)$, independent of the choice of $E_{1}$ and $B_{2}$.

\section{Representing the PRoduct of OUter INVERSES}

In this section, we assume that for two linear maps $T_{1}: V \rightarrow W$ and $T_{2}: U \rightarrow V$ with outer inverses $G_{1}$ and $G_{2}$ the reverse order law holds. Our goal is to find a description of the product $G_{2} G_{1}$ that does not require the explicit knowledge of $G_{1}$ and $G_{2}$. Using the representation via projectors, one immediately verifies that

$$
\mathrm{O}\left(T_{2}, P_{2}, Q_{2}\right) \mathrm{O}\left(T_{1}, P_{1}, Q_{1}\right)=\mathrm{O}\left(T_{1} T_{2}, P_{2}-G_{2} P_{1} T_{2}, T_{1} Q_{2} G_{1}\right)
$$

but this expression involves both outer inverses $G_{1}$ and $G_{2}$. For the representation via defining spaces, we compute the kernel and the image of the product.

Lemma 14. Let $T_{1}: V \rightarrow W$ and $T_{2}: U \rightarrow V$ be linear with outer inverses $G_{1}=$ $\mathrm{O}\left(T_{1}, B_{1}, E_{1}\right)$ and $G_{2}=\mathrm{O}\left(T_{2}, B_{2}, E_{2}\right)$. Then

$$
\operatorname{Ker} G_{2} G_{1}=E_{1} \oplus T_{1}\left(B_{1} \cap E_{2}\right) \quad \text { and } \quad \operatorname{Im} G_{2} G_{1}=G_{2}\left(\left(B_{1}+E_{2}\right) \cap \operatorname{Im} T_{2}\right) .
$$

Proof. Recall that by definition $\operatorname{Ker} G_{i}=E_{i}$ and $\operatorname{Im} G_{i}=B_{i}$. The first identity follows directly from Proposition 4. For the second identity, we first note that for a linear map $G$ and subspaces $V_{1}, V_{2}$, we have $G\left(V_{1} \cap V_{2}\right)=G\left(V_{1}\right) \cap G\left(V_{2}\right)$ if $\operatorname{Ker} G \leq V_{1}$. Hence $G_{2}\left(\left(B_{1}+E_{2}\right) \cap \operatorname{Im} T_{2}\right)$ equals

$$
G_{2}\left(\left(\operatorname{Im} G_{1}+\operatorname{Ker} G_{2}\right) \cap \operatorname{Im} T_{2}\right)=G_{2}\left(\operatorname{Im} G_{1}\right) \cap G_{2}\left(\operatorname{Im} T_{2}\right)=\operatorname{Im} G_{2} G_{1},
$$

since $G_{2}\left(\operatorname{Im} T_{2}\right)=\operatorname{Im} G_{2}$ by Proposition 2(ii),

Note that the expression for the image of the composition requires the explicit knowledge of $G_{2}$. In particular, the reverse order law takes the form

$\mathrm{O}\left(T_{2}, B_{2}, E_{2}\right) \mathrm{O}\left(T_{1}, B_{1}, E_{1}\right)=\mathrm{O}\left(T_{1} T_{2}, G_{2}\left(\left(B_{1}+E_{2}\right) \cap \operatorname{Im} T_{2}\right), E_{1}+T_{1}\left(B_{1} \cap E_{2}\right)\right)$.

Werner [28, Thm. 2.4] gives a result in a similar spirit for inner inverses of matrices.

Using an implicit description of $\operatorname{Im} G_{i}$, it is possible to state the reverse order law in a form that depends on the kernels and images of the respective outer inverses alone. This approach is motivated by our application to linear boundary problems 
(Section 9), where it is natural to define solution spaces via the boundary conditions they satisfy.

In more detail, the Galois connection from Appendix $\AA$ allows to represent a subspace $B$ implicitly via the orthogonally closed subspace $\mathscr{B}=B^{\perp}$ of the dual space. We will therefore use the notation $G=\mathrm{O}(T, \mathscr{B}, E)$ for the outer inverse with $\operatorname{Im} G=\mathscr{B}^{\perp}$ and $\operatorname{Ker} G=E$ as well as the analogue for inner inverses.

Theorem 15. Let $T_{1}: V \rightarrow W$ and $T_{2}: U \rightarrow V$ be linear with outer inverses $G_{1}=\mathrm{O}\left(T_{1}, \mathscr{B}_{1}, E_{1}\right)$ and $G_{2}=\mathrm{O}\left(T_{2}, \mathscr{B}_{2}, E_{2}\right)$. If $G_{2} G_{1}$ is an outer inverse of $T_{1} T_{2}$, then

(6) $\mathrm{O}\left(T_{2}, \mathscr{B}_{2}, E_{2}\right) \mathrm{O}\left(T_{1}, \mathscr{B}_{1}, E_{1}\right)=\mathrm{O}\left(T_{1} T_{2}, \mathscr{B}_{2} \oplus T_{2}^{*}\left(\mathscr{B}_{1} \cap E_{2}^{\perp}\right), E_{1} \oplus T_{1}\left(\mathscr{B}_{1}^{\perp} \cap E_{2}\right)\right)$, where $T_{2}^{*}$ denotes the transpose of $T_{2}$.

Proof. From Lemma 14 we already know that $\operatorname{Ker} G_{2} G_{1}=E_{1} \oplus T_{1}\left(\mathscr{B}_{1}^{\perp} \cap E_{2}\right)$. From Proposition 20 and 4 we get

$$
\begin{aligned}
\left(\operatorname{Im} G_{2} G_{1}\right)^{\perp} & =\operatorname{Ker} G_{1}^{*} G_{2}^{*}=T_{2}^{*}\left(\operatorname{Ker} G_{1}^{*} \cap \operatorname{Im} G_{2}^{*}\right) \oplus \operatorname{Ker} G_{2}^{*} \\
& =T_{2}^{*}\left(\left(\operatorname{Im} G_{1}\right)^{\perp} \cap\left(\operatorname{Ker} G_{2}\right)^{\perp}\right) \oplus\left(\operatorname{Im} G_{2}\right)^{\perp}=T_{2}^{*}\left(\mathscr{B}_{1} \cap E_{2}^{\perp}\right) \oplus \mathscr{B}_{2},
\end{aligned}
$$

and thus (6) holds.

A computational advantage of this representation is that one can determine $G_{2} G_{1}$ directly by computing only one outer inverse instead of computing both $G_{1}$ and $G_{2}$; see the next section for an example.

\section{EXAMPLES FOR MATRICES}

In this section, we illustrate our results for finite-dimensional vector spaces. In particular, we show how to compute directly the composition of two generalized inverses using the reverse order law in the form (6).

Consider the following linear maps $T_{1}: \mathbb{Q}^{4} \rightarrow \mathbb{Q}^{3}$ and $T_{2}: \mathbb{Q}^{3} \rightarrow \mathbb{Q}^{4}$ given by

$$
T_{1}=\left(\begin{array}{cccc}
1 & -1 & -1 & 1 \\
0 & 2 & 2 & -2 \\
3 & 1 & 1 & -1
\end{array}\right) \quad \text { and } \quad T_{2}=\left(\begin{array}{ccc}
1 & -2 & -1 \\
1 & 1 & 2 \\
-1 & 5 & 4 \\
-1 & 5 & 4
\end{array}\right)
$$

We first use Theorem 10 and 11 to check whether for algebraic generalized inverses $G_{1}=\mathrm{G}\left(T_{1}, B_{1}, E_{1}\right)$ and $G_{2}=\mathrm{G}\left(T_{2}, B_{2}, E_{2}\right)$ the composition $G_{2} G_{1}$ is an algebraic generalized inverse of $T_{1} T_{2}$.

For testing the conditions, we only need to fix $B_{1}=\operatorname{Im} G_{1}$ and $E_{2}=\operatorname{Ker} G_{2}$, such that $B_{1} \oplus \operatorname{Ker} T_{1}=\mathbb{Q}^{4}=E_{2} \oplus \operatorname{Im} T_{2}$. We have

$\operatorname{Ker} T_{1}=\operatorname{span}\left((0,1,0,1)^{T},(0,0,1,1)^{T}\right), \quad \operatorname{Im} T_{2}=\operatorname{span}\left((1,0,-2,-2)^{T},(0,1,1,1)^{T}\right)$, so we may choose for example

$$
B_{1}=\operatorname{span}\left((1,0,0,0)^{T},(0,1,0,0)^{T}\right), \quad E_{2}=\operatorname{span}\left((1,0,0,0)^{T},(0,0,1,0)^{T}\right) .
$$

For algebraic generalized inverses, we obtain as a necessary and sufficient condition for being an outer inverse

$$
B_{1} \leq \operatorname{Im} T_{2} \oplus\left(E_{2} \cap B_{1}\right) \oplus\left(E_{2} \cap \operatorname{Ker} T_{1}\right)
$$

from Theorem 10 (iii). 
Since $E_{2} \cap \operatorname{Ker} T_{1}=\{0\}$ and $E_{2} \cap B_{1}=\operatorname{span}\left((1,0,0,0)^{T}\right)$, the right hand side yields that $\operatorname{span}\left((1,0,0,0)^{T},(0,1,0,0)^{T},(0,0,1,1)^{T}\right) \geq B_{1}$. Thus for all algebraic generalized inverses $G_{1}$ and $G_{2}$ with $\operatorname{Im} G_{1}=B_{1}$ and $\operatorname{Ker} G_{2}=E_{2}$, the product $G_{2} G_{1}$ is an outer inverse of $T_{1} T_{2}$.

The corresponding condition for inner inverses by Theorem 11](iii) is

$$
\operatorname{Im} T_{2} \leq B_{1} \oplus\left(\operatorname{Ker} T_{1} \cap \operatorname{Im} T_{2}\right) \oplus\left(\operatorname{Ker} T_{1} \cap E_{2}\right) .
$$

Since $\operatorname{Ker} T_{1} \cap \operatorname{Im} T_{2}=\{0\}$, the right hand side yields $B_{1}$, which does not contain $\operatorname{Im} T_{2}$. Hence for the above choices of $G_{1}$ and $G_{2}$, the product $G_{2} G_{1}$ is never an inner inverse of $T_{1} T_{2}$.

Since $G_{2} G_{1}$ is an outer inverse, Theorem [15] allows to determine $G_{2} G_{1}$ directly without knowing the factors. Identifying the dual space with row vectors, the orthogonals of $B_{1}$ and $E_{2}$ are given by

$$
B_{1}^{\perp}=\mathscr{B}_{1}=\operatorname{span}((0,0,1,0),(0,0,0,1)), \quad E_{2}^{\perp}=\operatorname{span}((0,1,0,0),(0,0,0,1)),
$$

so we have $\mathscr{B}_{1}^{\perp} \cap E_{2}=\operatorname{span}\left((1,0,0,0)^{T}\right)$ and $\mathscr{B}_{1} \cap E_{2}^{\perp}=\operatorname{span}((0,0,0,1))$. For explicitly computing $G_{2} G_{1}$, we also have to choose $B_{2}=\operatorname{Im} G_{2}$ and $E_{1}=\operatorname{Ker} G_{1}$. Since we have

$$
\left.\operatorname{Im} T_{1}=\operatorname{span}\left((1,0,3)^{T}\right),(0,1,2)^{T}\right), \quad \operatorname{Ker} T_{2}=\operatorname{span}\left((1,1,-1)^{T}\right),
$$

we may choose the complements $E_{1}=\operatorname{Ker} G_{1}$ and $B_{2}=\operatorname{Im} G_{2}$ as

$$
E_{1}=\operatorname{span}\left((0,0,1)^{T}\right) \quad \text { and } \quad B_{2}=\operatorname{span}\left((1,0,0)^{T},(0,1,0)^{T}\right) .
$$

Using (6), we can determine the kernel

$$
E=\operatorname{Ker} G_{2} G_{1}=E_{1} \oplus T_{1}\left(\mathscr{B}_{1}^{\perp} \cap E_{2}\right)=\operatorname{span}\left((1,0,0)^{T},(0,0,1)^{T}\right) .
$$

The image of $G_{2} G_{1}$ is by (6) given via the orthogonal

$$
\left(\operatorname{Im} G_{2} G_{1}\right)^{\perp}=\mathscr{B}_{2} \oplus T_{2}^{*}\left(\mathscr{B}_{1} \cap E_{2}^{\perp}\right)=\operatorname{span}((0,0,1),(-1,5,4)),
$$

which means that $B=\operatorname{Im} G_{2} G_{1}=\operatorname{span}\left((5,1,0)^{T}\right)$. Therefore we can directly determine $G$ as the unique outer inverse

$$
G=\mathrm{O}\left(T_{1} T_{2}, B, E\right)=\left(\begin{array}{ccc}
0 & \frac{5}{12} & 0 \\
0 & \frac{1}{12} & 0 \\
0 & 0 & 0
\end{array}\right) .
$$

One easily checks that $G$ is an outer inverse of $T$.

\section{Fredholm operators}

We now turn to algorithmic aspects of the previous results. As already emphasized, for arbitrary vector spaces we can express conditions for the reverse order law in terms of the defining spaces alone. Nevertheless, in general it will not be possible to compute sums and intersections of infinite-dimensional subspaces. For algorithmically checking the conditions of Theorem 10 or 11 and for computing the reverse order law in the form (6), we consider finite (co)dimensional spaces and Fredholm operators.

Recall that a linear map $T$ between vector spaces is called Fredholm operator if $\operatorname{dim} \operatorname{Ker} T<\infty$ and codim $\operatorname{Im} T<\infty$. Moreover, for finite codimensional subspaces $V_{1} \leq V$, we have codim $V_{1}=\operatorname{dim} V_{1}^{\perp}$. In this case, $V_{1}$ can be implicitly represented by the finite-dimensional subspace $V_{1}^{\perp} \leq V^{*}$. For an application to linear ordinary boundary problems, see the next section. 
We assume that for finite-dimensional subspaces, we can compute sums and intersections and check inclusions, both in vector spaces and in their duals. With the following lemma, the intersection of a finite-dimensional subspace with a finite codimensional subspace is reduced to computing kernels of matrices.

Definition 16. Let $u=\left(u_{1}, \ldots, u_{m}\right)^{T} \in V^{m}$ and $\beta=\left(\beta_{1}, \ldots, \beta_{n}\right)^{T} \in\left(V^{*}\right)^{n}$. We call

$$
\beta(u)=\left(\begin{array}{ccc}
\beta_{1}\left(u_{1}\right) & \ldots & \beta_{1}\left(u_{m}\right) \\
\vdots & \ddots & \vdots \\
\beta_{n}\left(u_{1}\right) & \ldots & \beta_{n}\left(u_{m}\right)
\end{array}\right) \in F^{n \times m}
$$

the evaluation matrix of $\beta$ and $u$.

Lemma 17. Let $U \leq V$ and $\mathscr{B} \leq V^{*}$ be generated respectively by $u=\left(u_{1}, \ldots, u_{m}\right)$ and $\beta=\left(\beta_{1}, \ldots, \beta_{n}\right)$. Let $k^{1}, \ldots, k^{r} \in F^{m}$ be a basis of $\operatorname{Ker} \beta(u)$, and $\kappa^{1}, \ldots, \kappa^{s} \in$ $F^{n}$ a basis of $\operatorname{Ker}(\beta(u))^{T}$. Then

(i) $U \cap \mathscr{B}^{\perp}$ is generated by $\sum_{i=1}^{m} k_{i}^{1} u_{i}, \ldots, \sum_{i=1}^{m} k_{i}^{r} u_{i}$ and

(ii) $U^{\perp} \cap \mathscr{B}$ is generated by $\sum_{i=1}^{n} \kappa_{i}^{1} \beta_{i}, \ldots, \sum_{i=1}^{n} \kappa_{i}^{s} \beta_{i}$.

Proof. A linear combination $v=\sum_{\ell=1}^{m} c_{\ell} u_{\ell}$ is in $\mathscr{B}^{\perp}$ if and only if $\beta_{i}(v)=0$ for $1 \leq i \leq n$, that is, $\sum_{\ell=1}^{m} c_{\ell} \beta_{i}\left(u_{\ell}\right)=0$ for $1 \leq i \leq n$. Hence $\beta(u) \cdot\left(c_{1}, \ldots, c_{m}\right)^{T}=0$. Analogously, one sees that the coefficients of linear combination in $U^{\perp} \cap \mathscr{B}$ are in the kernel of $(\beta(u))^{T}$.

We reformulate the conditions of Theorem 10 such that for Fredholm operators they only involve operations on finite-dimensional subspaces and intersections like in the previous lemma. Similarly, one can rewrite the conditions of Theorem 11.

Corollary 18. Let $T_{1}: V \rightarrow W$ and $T_{2}: U \rightarrow V$ be linear with outer inverses $G_{1}=$ $\mathrm{O}\left(T_{1}, \mathscr{B}_{1}, E_{1}\right)$ and $G_{2}=\mathrm{O}\left(T_{2}, \mathscr{B}_{2}, E_{2}\right)$. Let $\mathscr{C}_{2}=T_{2}\left(\mathscr{B}_{2}^{\perp}\right)^{\perp}$ and $K_{1}=T_{1}^{-1}\left(E_{1}\right)$. The following conditions are equivalent:

(i) $G_{2} G_{1}$ is an outer inverse of $T_{1} T_{2}$.

(ii) $\mathscr{C}_{2}+\left(\mathscr{B}_{1} \cap E_{2}^{\perp}\right) \geq \mathscr{B}_{1} \cap\left(E_{2} \cap K_{1}\right)^{\perp}$

(iii) $\mathscr{B}_{1} \geq \mathscr{C}_{2} \cap\left(E_{2} \cap \mathscr{B}_{1}^{\perp}\right)^{\perp} \cap\left(E_{2} \cap K_{1}\right)^{\perp}$

(iv) $K_{1} \oplus\left(E_{2} \cap \mathscr{B}_{1}^{\perp}\right) \geq E_{2} \cap\left(\mathscr{B}_{1} \cap \mathscr{C}_{2}\right)^{\perp}$

(v) $E_{2} \geq K_{1} \cap\left(\mathscr{B}_{1} \cap E_{2}^{\perp}\right)^{\perp} \cap\left(\mathscr{B}_{1} \cap \mathscr{C}_{2}\right)^{\perp}$

Proof. Taking the orthogonal of both sides of 10 (ii) (iii) respectively and applying Proposition [19 we get (ii) and (iii). For (iv) and (v) we can apply Proposition 19 directly to the corresponding conditions of Theorem 10.

We note that using Lemma 17, it also possible to determine constructively the implicit representation (6) of a product of generalized inverses; see the next section.

\section{EXAMPLES FOR LINEAR ORDINARY BOUNDARY PROBLEMS}

As an example involving infinite dimensional spaces and Fredholm operators, we consider solution (Green's) operators for linear ordinary boundary problems. Algebraically, linear boundary problems can be represented as a pair $(T, \mathscr{B})$, where $T: V \rightarrow W$ is a surjective linear map, and $\mathscr{B} \leq V^{*}$ is an orthogonally closed subspace of (homogeneous) boundary conditions. We say that $v \in V$ is a solution of $(T, \mathscr{B})$ for a given $w \in W$ if $T v=w$ and $v \in \mathscr{B}^{\perp}$. 
For a regular boundary problem (having a unique solution for every right-hand side), the Green's operator is defined as the unique right inverse $G$ of $T$ with $\operatorname{Im} G=\mathscr{B}^{\perp}$; see [20] for further details. The product $G_{2} G_{1}$ of the Green's operators of two boundary problems $\left(T_{1}, \mathscr{B}_{1}\right)$ and $\left(T_{2}, \mathscr{B}_{2}\right)$ is then the Green's operator of the regular boundary problem $\left(T_{1} T_{2}, \mathscr{B}_{2} \oplus T_{2}^{*}\left(\mathscr{B}_{1}\right)\right)$, see also Theorem 15 .

For boundary problems having at most one solution, that is $\mathscr{B}^{\perp} \cap \operatorname{Ker} T=\{0\}$, the linear algebraic setting has been extended in 12 by defining generalized Green's operators as outer inverses. More specifically, one first has to project an arbitrary right-hand side $w \in W$ onto $T\left(\mathscr{B}^{\perp}\right)$, the image of the "functions" satisfying the boundary conditions, along a complement $E$ of $T\left(\mathscr{B}^{\perp}\right)$. The corresponding generalized Green's operator is defined as the outer inverse $G=\mathrm{O}(T, \mathscr{B}, E)$, and we refer to $E \leq W$ as an exceptional space for the boundary problem $(T, \mathscr{B})$.

The question when the product of two outer inverses is again an outer inverse, is the basis for factoring boundary problems into lower order problems; see [20, 21] for the case of regular boundary problems. This, in turn, provides a method to factor certain integral operators.

As an example, let us consider the boundary problem

$$
\begin{aligned}
& u^{\prime \prime}=f \\
& u^{\prime}(0)=u^{\prime}(1)=u(1)=0
\end{aligned}
$$

In the above setting, this means we consider the pair $\left(T_{1}, \mathscr{B}_{1}\right)$ with $T_{1}=\mathrm{D}^{2}$ and $\mathscr{B}_{1}=\operatorname{span}\left(\mathrm{E}_{0} \mathrm{D}, \mathrm{E}_{1} \mathrm{D}, \mathrm{E}_{1}\right)$, where $\mathrm{D}$ denotes the usual derivation on smooth functions and $\mathrm{E}_{c}$ the evaluation at $c \in \mathbb{R}$. The boundary problem is only solvable for forcing functions $f$ satisfying the compatibility condition $\int_{0}^{1} f(\xi) \mathrm{d} \xi=0$; more abstractly, we have $T_{1}\left(\mathscr{B}_{1}^{\perp}\right)=\mathscr{C}_{1}^{\perp}$ with $\mathscr{C}_{1}=\operatorname{span}\left(\int_{0}^{1}\right)$, where $\int_{0}^{1}$ denotes the functional $f \mapsto \int_{0}^{1} f(\xi) \mathrm{d} \xi$. For computing a generalized Green's operator of $\left(T_{1}, \mathscr{B}_{1}, E_{1}\right)$, we have to project $f$ onto $\mathscr{C}_{1}^{\perp}$ along a fixed complement $E_{1}$. In [13, we computed the generalized Green's operator

$$
G_{1}(f)=x \int_{0}^{x} f(\xi) \mathrm{d} \xi-\int_{0}^{x} \xi f(\xi) \mathrm{d} \xi-\frac{1}{2}\left(x^{2}+1\right) \int_{0}^{1} f(\xi) \mathrm{d} \xi+\int_{0}^{1} \xi f(\xi) \mathrm{d} \xi
$$

of (77) for $E_{1}=\mathbb{R}$ being the constant functions. It is easy to see that in this case we have $T_{1}^{-1}\left(E_{1}\right)=\operatorname{span}\left(1, x, x^{2}\right)$.

As a second boundary problem, we consider

$$
\begin{aligned}
& u^{\prime \prime}-u=f \\
& u^{\prime}(0)=u^{\prime}(1)=u(1)=0
\end{aligned}
$$

or $\left(T_{2}, \mathscr{B}_{2}\right)$ with $T_{2}=\mathrm{D}^{2}-1$ and $\mathscr{B}_{2}=\operatorname{span}\left(\mathrm{E}_{0} \mathrm{D}, \mathrm{E}_{1} \mathrm{D}, \mathrm{E}_{1}\right)$. For the corresponding generalized Green's operator $G_{2}$ with exceptional space $E_{2}=\operatorname{span}(x)$, we will now check if the products $G_{1} G_{2}$ and $G_{2} G_{1}$ are again generalized Green's operators of $T_{1} T_{2}=T_{2} T_{1}=\mathrm{D}^{4}-\mathrm{D}^{2}$, using condition (ii) of Corollary 18

We use the algorithm from [13, implemented in the package IntDiffOp for the computer algebra system MAPLE, to compute the compatibility conditions. The algorithm is based on the identity

$$
T\left(\mathscr{B}^{\perp}\right)^{\perp}=G^{*}\left(\mathscr{B} \cap(\operatorname{Ker} T)^{\perp}\right),
$$

for any right inverse $G$ of $T$, which follows from Propositions 20 and 4 . Moreover, a right inverse of the differential operator can be computed by variation of 
constants and the intersection $\mathscr{B} \cap(\operatorname{Ker} T)^{\perp}$ using Lemma 17. Thus we obtain $\mathscr{C}_{2}=\operatorname{span}\left(\int_{0}^{1}(\exp (-x)+\exp (x))\right)$, where $\int_{0}^{1}(\exp (-x)+\exp (x))$ denotes the functional $f \mapsto \int_{0}^{1}(\exp (-\xi)+\exp (\xi)) f(\xi) \mathrm{d} \xi$.

The space $T_{2}^{-1}\left(E_{2}\right)=\operatorname{span}(x, \exp (x), \exp (-x))$ can be computed using Proposition 4 and a right inverse of the differential operator; this is also implemented in the IntDiffOp package. Hence we have $E_{1} \cap T_{2}^{-1}\left(E_{2}\right)=\{0\}$ and therefore $\mathscr{B}_{2} \cap\left(E_{1} \cap T_{2}^{-1}\left(E_{2}\right)\right)^{\perp}=\mathscr{B}_{2}$. Computing $\mathscr{B}_{2} \cap E_{1}^{\perp}$ with Lemma 17]yields $\mathscr{B}_{2} \cap E_{1}^{\perp}=$ $\operatorname{span}\left(\mathrm{E}_{0} \mathrm{D}, \mathrm{E}_{1} \mathrm{D}\right)$; thus $G_{1} G_{2}$ is not an outer inverse of the product $T_{2} T_{1}=\mathrm{D}^{4}-\mathrm{D}^{2}$ by Corollary 18 (ii).

On the other hand, we have $E_{2} \cap T_{1}^{-1}\left(E_{1}\right)=\operatorname{span}(x)=E_{2}$, hence we know by Corollary 18 (ii) that $G_{2} G_{1}$ is an outer inverse of $T_{1} T_{2}=\mathrm{D}^{4}-\mathrm{D}^{2}$. Furthermore, by Theorem 15 we can determine which boundary problem it solves without computing $G_{1}$ and $G_{2}$. With Lemma 17 we obtain $\mathscr{B}_{1}^{\perp} \cap E_{2}=\{0\}$ and $\mathscr{B}_{1} \cap E_{2}^{\perp}=\operatorname{span}\left(\mathrm{E}_{0} \mathrm{D}-\mathrm{E}_{1}, \mathrm{E}_{1} \mathrm{D}-\mathrm{E}_{1}\right)$. Since applying the transpose $T_{2}^{*}$ to $\mathrm{E}_{0} \mathrm{D}-\mathrm{E}_{1}$ and $\mathrm{E}_{1} \mathrm{D}-\mathrm{E}_{1}$ corresponds to multiplying $T_{2}=\mathrm{D}^{2}-1$ from the right, $G_{2} G_{1}$ is the generalized Green's operator of

$$
\left(D^{4}-D^{2}, \operatorname{span}\left(E_{0} D, E_{1} D, E_{1}, E_{0} D^{3}-E_{1} D^{2}, E_{1} D^{3}-E_{1} D^{2}\right), \mathbb{R}\right)
$$

by (6); or, in traditional notation, it solves the boundary problem

$$
\begin{aligned}
& u^{\prime \prime \prime \prime}-u^{\prime \prime}=f \\
& u^{\prime}(0)=u^{\prime}(1)=u(1)=u^{\prime \prime \prime}(0)-u^{\prime \prime}(1)=u^{\prime \prime \prime}(1)-u^{\prime \prime}(1)=0,
\end{aligned}
$$

with exceptional space $\mathbb{R}$.

\section{ACKNOWLEDGEMENTS}

We would like to thank an anonymous referee for his detailed comments. G.R. was supported by the Austrian Science Fund (FWF): J 3030-N18.

\section{Appendix A. Duality}

In the appendix, we summarize duality results for arbitrary vector spaces and their duals that generalize the standard duality for finite-dimensional vector spaces but do not require any topological assumptions; see [14, Section 9.2 and 9.3] and [20] for further details. The notation should also remind of the analogous and well-known results for Hilbert spaces.

Let $V$ and $W$ be vector spaces over a field $F$ and $\langle\rangle:, V \times W \rightarrow F$ be a bilinear map. For $V_{1} \leq V$, we define the orthogonal

$$
V_{1}^{\perp}=\left\{w \in W \mid\langle v, w\rangle=0 \text { for all } v \in V_{1}\right\} \leq W .
$$

The orthogonal $W_{1}^{\perp}$ for $W_{1} \leq W$ is defined analogously. A subspace $U$ is called orthogonally closed if $U=U^{\perp \perp}$. It follows directly from the definition that for all subsets $X_{1}, X_{2} \subseteq V$, we have $X_{1} \subseteq X_{2} \Rightarrow X_{1}^{\perp} \supseteq X_{2}^{\perp}$ and $X_{1} \subseteq X_{1}^{\perp \perp}$; and the same holds for subsets of $W$. Let $\mathbb{P}(V)$ denote the projective geometry of $V$, that is, the partially ordered set (poset) of all subspaces ordered by inclusion. Then we have an order-reversing Galois connection between $\mathbb{P}(V)$ and $\mathbb{P}(W)$ defined by $U \mapsto U^{\perp}$.

We now consider the canonical bilinear form $V \times V^{*} \rightarrow F$ of a vector space $V$ and its dual $V^{*}$ defined by $\langle v, \beta\rangle \mapsto \beta(v)$. Then every subspace $V_{1} \leq V$ is orthogonally closed with respect to the canonical bilinear form, and every finite-dimensional 
subspace $\mathscr{B} \leq V^{*}$ is orthogonally closed. The Galois connection gives an orderreversing bijection between $\mathbb{P}(V)$ and the poset of all orthogonally closed subspaces of $V^{*}$. So we can describe any subspace $V_{1} \leq V$ implicitly by the corresponding orthogonally closed subspace $V_{1}^{\perp}$. We denote the poset of all orthogonally closed subspaces of $V^{*}$ with $\overline{\mathbb{P}}\left(V^{*}\right)$.

The projective geometry $\mathbb{P}(V)$ is a modular lattice, where join and meet are defined as the sum and intersection of subspaces. Modularity means that for all $V_{1}, V_{2}, V_{3} \in \mathbb{P}(V)$ with $V_{1} \leq V_{3}$ we have

$$
V_{1}+\left(V_{2} \cap V_{3}\right)=\left(V_{1}+V_{2}\right) \cap V_{3} .
$$

Moreover, for spaces $V_{1} \leq V_{3}$ and $V_{2} \leq V_{4}$, we have

$$
V=V_{1}+V_{2}=V_{3} \oplus V_{4} \Rightarrow V_{1}=V_{3} \text { and } V_{2}=V_{4},
$$

since $V_{3} \cap V_{4}=\{0\}$ implies $V_{3}=\left(V_{1} \oplus V_{2}\right) \cap V_{3}=V_{1}$ and $V_{4}=\left(V_{1} \oplus V_{2}\right) \cap V_{4}=V_{2}$.

One can also show that $\overline{\mathbb{P}}\left(V^{*}\right)$ is a modular lattice, where the meet is the intersection and the join is the orthogonal closure of the sum of subspaces. Using this fact, one can prove in particular that the sum of two orthogonally closed subspaces is orthogonally closed. The following theorem summarizes Section 9.3 of [14.

Proposition 19. The map $V_{1} \mapsto V_{1}^{\perp}$ gives an order-reversing lattice isomorphism with inverse $\mathscr{B}_{1} \mapsto \mathscr{B}_{1}^{\perp}$ between the complemented modular lattices $\mathbb{P}(V)$ and $\overline{\mathbb{P}}\left(V^{*}\right)$. In particular, the intersection of orthogonally closed subspaces in $V^{*}$ is orthogonally closed and

$$
\left(V_{1}+V_{2}\right)^{\perp}=V_{1}^{\perp} \cap V_{2}^{\perp} \quad \text { and } \quad\left(\mathscr{B}_{1} \cap \mathscr{B}_{2}\right)^{\perp}=\mathscr{B}_{1}^{\perp}+\mathscr{B}_{2}^{\perp} .
$$

The sum of two orthogonally closed subspaces in $V^{*}$ is orthogonally closed and

$$
\left(V_{1} \cap V_{2}\right)^{\perp}=V_{1}^{\perp}+V_{2}^{\perp} \text { and }\left(\mathscr{B}_{1}+\mathscr{B}_{2}\right)^{\perp}=\mathscr{B}_{1}^{\perp} \cap \mathscr{B}_{2}^{\perp} .
$$

Furthermore, orthogonality preserves direct sums, such that

$$
V=V_{1} \oplus V_{2} \Rightarrow V^{*}=V_{1}^{\perp} \oplus V_{2}^{\perp} \quad \text { and } \quad V^{*}=\mathscr{B}_{1} \oplus \mathscr{B}_{2} \Rightarrow V=\mathscr{B}_{1}^{\perp} \oplus \mathscr{B}_{2}^{\perp} .
$$

For a linear map $A: V \rightarrow W$ between vector spaces, the transpose $A^{*}: W^{*} \rightarrow V^{*}$ is defined by $\gamma \mapsto \gamma \circ A$. The transposition map $A \mapsto A^{*}$ from $L(V, W)$ to $L\left(W^{*}, V^{*}\right)$ is linear, and it is injective since for all $w \neq 0$ there exists a linear map $h \in W^{*}$ with $h(w) \neq 0$. Moreover, the transpose of a composition is given by $\left(A_{1} A_{2}\right)^{*}=A_{2}^{*} A_{1}^{*}$.

The image of an orthogonally closed space under the transpose map is orthogonally closed, and we have following identities, see, for example, [20, Prop. A.6].

Proposition 20. Let $V$ and $W$ be vector spaces and $A: V \rightarrow W$ be linear. Then

$$
\begin{aligned}
A\left(V_{1}\right)^{\perp} & =\left(A^{*}\right)^{-1}\left(V_{1}^{\perp}\right), & A\left(\mathscr{B}_{1}^{\perp}\right) & =\left(A^{*}\right)^{-1}\left(\mathscr{B}_{1}\right)^{\perp}, \\
A^{*}\left(\mathscr{C}_{1}\right)^{\perp} & =A^{-1}\left(\mathscr{C}_{1}^{\perp}\right), & A^{*}\left(W_{1}^{\perp}\right) & =A^{-1}\left(W_{1}\right)^{\perp},
\end{aligned}
$$

for subspaces $V_{1} \leq V, W_{1} \leq W, \mathscr{C}_{1} \leq W^{*}$ and orthogonally closed subspaces $\mathscr{B}_{1} \leq V^{*}$. In particular,

$(\operatorname{Im} A)^{\perp}=\operatorname{Ker} A^{*}, \quad \operatorname{Im} A=\left(\operatorname{Ker} A^{*}\right)^{\perp}, \quad\left(\operatorname{Im} A^{*}\right)^{\perp}=\operatorname{Ker} A, \quad \operatorname{Im} A^{*}=(\operatorname{Ker} A)^{\perp}$, for the image and kernel of $A$ and $A^{*}$.

The property of being a projector, outer/inner/algebraic generalized inverse carries over to the transpose. 
Proposition 21. A linear map $P: V \rightarrow V$ is a projector if and only if its transpose $P^{*}$ is a projector. A linear map $G: W \rightarrow V$ is an outer/inner/algebraic generalized inverse of $T: V \rightarrow W$ if and only if $G^{*}$ is an outer/inner/algebraic generalized inverse of $T^{*}$.

Proof. This follows from the defining equations for these properties. For example, if $G$ is an outer inverse of $T$, we have $G^{*} T^{*} G^{*}=(G T G)^{*}=G^{*}$, and the reverse implication follows from the injectivity of the transposition map.

With the results of this section, we obtain the following duality principle for generalized inverses.

Remark 22. Given a valid statement for linear maps on arbitrary vector spaces $V$ over a common field involving inclusions, $\{0\}$ and $V$, sums and intersections, direct sums, kernels and images, projectors, and outer/inner/algebraic generalized inverses, we obtain a valid dual statement by

- reversing the order of the linear maps and the corresponding domains and codomains,

- reversing inclusions and interchanging $V$ and $\{0\}$,

- interchanging sums and intersections,

- interchanging kernels and images.

For example, one easily checks that in Proposition 2, the statements (v) (vii) are the duals of (ii) (iv) in this sense.

\section{REFERENCES}

[1] Jerzy K. Baksalary and Oskar Maria Baksalary, Commutativity of projectors, Linear Algebra Appl. 341 (2002), 129-142.

[2] Adi Ben-Israel and Thomas N. E. Greville, Generalized inverses, second ed., Springer-Verlag, New York, 2003.

[3] A. L. Brown and A. Page, Elements of functional analysis, Van Nostrand Reinhold Company, London-New York-Toronto, Ont., 1970.

[4] A. R. De Pierro and Musheng Wei, Reverse order law for reflexive generalized inverses of products of matrices, Linear Algebra Appl. 277 (1998), 299-311.

[5] Nebojša Č. Dinčić and Dragan S. Djordjević, Basic reverse order law and its equivalencies, Aequationes Math. 85 (2013), 505-517.

[6] D. Djordjević and Nebojša C Dinčić, Reverse order law for the Moore-Penrose inverse, J. Math. Anal. Appl. 361 (2010), 252-261.

[7] Dragan S. Djordjević and Vladimir Rakočević, Lectures on generalized inverses, University of Niš, Faculty of Sciences and Mathematics, Niš, 2008.

[8] Ivan Erdelyi, On the "reverse order law" related to the generalized inverse of matrix products, J. ACM 13 (1966), 439-443.

[9] T. N. E. Greville, Note on the generalized inverse of a matrix product, SIAM Rev. 8 (1966), $518-521$.

[10] Jürgen Groß, Some remarks concerning the reverse order law, Discuss. Math. Algebra Stochastic Methods 17 (1997), 135-141.

[11] Jürgen Groß and Götz Trenkler, On the product of oblique projectors, Linear Multilinear Algebra 44 (1998), 247-259.

[12] Anja Korporal, Symbolic Methods for Generalized Green's Operators and Boundary Problems, Ph.D. thesis, University of Linz, 2012.

[13] Anja Korporal, Georg Regensburger, and Markus Rosenkranz, Regular and singular boundary problems in Maple, Proceedings of CASC 2011 (Computer Algebra in Scientific Computing) (Berlin / Heidelberg) (Vladimir Gerdt, Wolfram Koepf, Ernst Mayr, and Evgenii Vorozhtsov, eds.), LNCS, vol. 6885, Springer, 2011, pp. 280-293.

[14] Gottfried Köthe, Topological vector spaces (volume I), Springer, New York, 1969. 
[15] Qiaohua Liu and Musheng Wei, Reverse order law for least squares g-inverses of multiple matrix products, Linear Multilinear Algebra 56 (2008), 491-506.

[16] Sujit Kumar Mitra, P. Bhimasankaram, and Saroj B. Malik, Matrix partial orders, shorted operators and applications, World Scientific Publishing Co. Pte. Ltd., Hackensack, NJ, 2010.

[17] M.Z. Nashed, Inner, outer, and generalized inverses in Banach and Hilbert spaces, Numer. Funct. Anal. and Optimiz. 9 (1987), 261-325.

[18] M.Z. Nashed and G.F. Vortuba, A unified operator theory of generalized inverses, Generalized Inverses and Applications (M. Z. Nashed, ed.), Academic Press, New York, 1976, pp. 1-109.

[19] C. Radhakrishna Rao and Sujit Kumar Mitra, Generalized inverse of matrices and its applications, John Wiley \& Sons Inc., New York, 1971.

[20] Georg Regensburger and Markus Rosenkranz, An algebraic foundation for factoring linear boundary problems, Ann. Mat. Pura Appl. (4) 188 (2009), 123-151.

[21] Markus Rosenkranz and Georg Regensburger, Solving and factoring boundary problems for linear ordinary differential equations in differential algebras, J. Symbolic Comput. 43 (2008), $515-544$.

[22] S. R. Searle, Linear models, John Wiley \& Sons Inc., New York, 1971.

[23] Nobuo Shinozaki and Masaaki Sibuya, The reverse order law $(A B)^{-}=B^{-} A^{-}$, Linear Algebra Appl. 9 (1974), 29-40.

[24] _ Further results on the reverse-order law, Linear Algebra Appl. 27 (1979), 9-16.

[25] Y. Takane and H. Yanai, On oblique projectors, Linear Algebra Appl. 289 (1999), 297-310.

[26] Yoshio Takane, Yongge Tian, and Haruo Yanai, On reverse-order laws for least-squares ginverses and minimum norm g-inverses of a matrix product, Aequationes Math. 73 (2007), $56-70$.

[27] Yongge Tian and Shizhen Cheng, Some identities for Moore-Penrose inverses of matrix products, Linear Multilinear Algebra 52 (2004), 405-420.

[28] Hans Joachim Werner, G-inverses of matrix products, Data Analysis and Statistical Inference (Bergisch Gladbach) (G. Trenkler S. Schach, ed.), Eul-Verlag, 1992, pp. 531-546.

[29] When is $B^{-} A^{-}$a generalized inverse of AB?, Linear Algebra Appl. 210 (1994), $255-263$.

[30] E.A. Wibker, R.B. Howe, and J.D. Gilbert, Explicit solutions to the reverse order law $(A B)^{+}=B_{m r}^{-} A_{l r}^{-}$, Linear Algebra Appl. 25 (1979), 107-114.

Johann Radon Institute for Computational and Applied Mathematics (RICAM), Austrian ACademy of Sciences, A-4040 Linz, Austria

E-mail address: anja.korporal@ricam.oeaw.ac.at

Johann Radon Institute for Computational and Applied Mathematics (RICAM), Austrian ACAdemy of Sciences, A-4040 Linz, Austria

E-mail address: georg.regensburger@ricam.oeaw.ac.at 